\title{
Les collections universitaires, un enjeu pour une nouvelle visibilité du campus bordelais
}

Sophie Chave-Dartoen, Lætitia Maison-Soulard et Marion Lagrange

\section{(2) OpenEdition \\ Journals}

Édition électronique

URL : http://journals.openedition.org/ocim/1255

DOI : $10.4000 /$ ocim. 1255

ISSN : 2108-646X

Éditeur

OCIM

Édition imprimée

Date de publication : 10 juillet 2013

Pagination : 21-31

ISSN : 0994-1908

Référence électronique

Sophie Chave-Dartoen, Lætitia Maison-Soulard et Marion Lagrange, "Les collections universitaires, un enjeu pour une nouvelle visibilité du campus bordelais », La Lettre de I'OCIM [En ligne], 148 | 2013, mis en ligne le 01 juillet 2015, consulté le 01 mai 2019. URL : http://journals.openedition.org/ocim/1255 DOI : $10.4000 /$ ocim. 1255

Ce document a été généré automatiquement le 1 mai 2019.

Tous droits réservés 


\title{
Les collections universitaires, un enjeu pour une nouvelle visibilité du campus bordelais
}

\author{
Sophie Chave-Dartoen, Lætitia Maison-Soulard et Marion Lagrange
}

1 Les collections universitaires, souvent méconnues du grand public comme de l'institution publique, sont aujourd'hui appréciées d'un œil nouveau grâce à une dynamique internationale, particulièrement perceptible au niveau européen ${ }^{1}$. En France, la mise en place des PRES (pôles de recherche et d'enseignement supérieur), de la loi LRU, ou encore du Plan Campus déployé par le ministère de l'Enseignement supérieur et de la Recherche, poussent à la construction ou à la réaffirmation d'une identité forte des universités. Celles-ci souhaitent désormais soutenir une politique culturelle spécifique à chacune et ouvrir l'institution sur la cité. Les collections universitaires peuvent largement y contribuer. En permettant de remonter la filiation dans laquelle la recherche con temporaine s'inscrit et d'expliquer la particularité des savoir-faire développés localement, elles forment in fine les racines de l'innovation. Ce socle commun de ressources sur lequel s'appuie la culture scientifique des universitaires, en regard de ses traditions universelles, nationales et locales, constitue, avec les fonds des bibliothèques, un véritable learning center. 


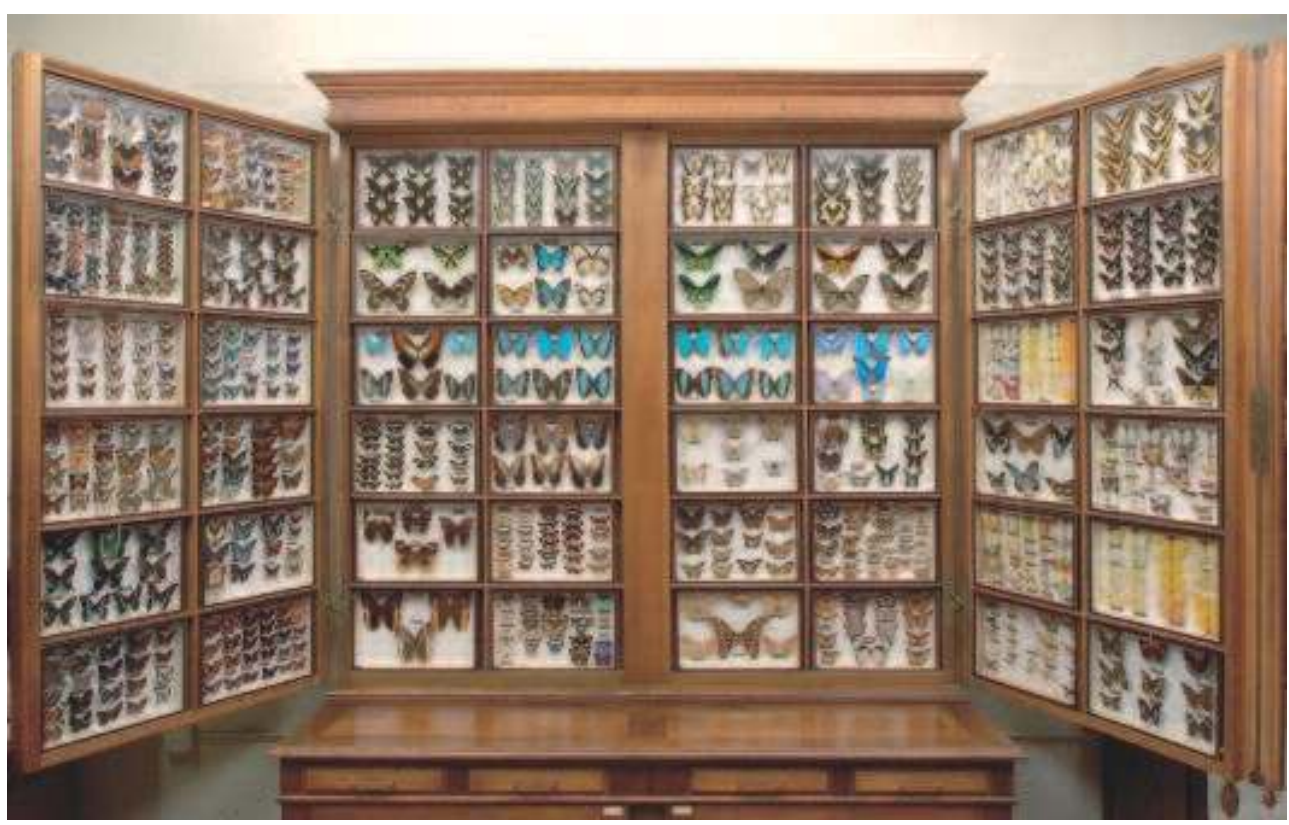

(C) Université Bordeaux

2 À Bordeaux, les différentes structures universitaires sont réunies depuis 2007 dans un PRES. En janvier 2014, l'université Bordeaux 1 (sciences et techniques), l'université Bordeaux Segalen (santé et sciences humaines et sociales) et l'université Bordeaux 4 Mon tesquieu (droit, science politique, économie) formeront la «Nouvelle université de Bordeaux » (NUB) ${ }^{2}$.

3 Dans ce contexte, le patrimoine universitaire bordelais, qui n'a jamais été doté d'un service gestionnaire à part entière, pourrait être vu comme un atout pour contribuer à forger un " esprit de campus » propre à la NUB et susciter la mise en place d'un projet global porté par une équipe.

4 La situation locale parait en effet propice. Différents programmes de recherche et de valorisation sont en cours depuis plusieurs années tandis qu'un travail de fond est entrepris, localement, afin de bien dégager les enjeux politiques et culturels d'un tel potentiel. Plusieurs actions ont été menées afin de sensibiliser les décideurs, la communauté universitaire et le grand public. Après un aperçu de la situation hétérogène des collections bordelaises, nous proposons ici une présentation des principales dynamiques en cours, que le projet de plateforme, soumis au bureau du PRES en septembre 2011, viendra compléter.

\section{État des lieux : une situation hétérogène}

Intimement liées au développement et à l'apprentissage des disciplines universitaires, les collections sont par nature diverses. L'histoire de l'université bordelaise, entre transformation de l'institution et création de nouveaux bâtiments, n'a pas toujours permis de conserver une cohérence en lien avec les espaces d'origine, voire avec les pratiques disciplinaires d'enseignement et de recherche. Mis à part les fonds patrimoniaux des bibliothèques universitaires, les collections scientifiques et 
pédagogiques sont dispersées entre les universités Bordeaux 1 (collections scientifiques et instrumentation), Bordeaux Segalen (ethnographie, botanique, instruments scientifiques, et quelques œuvres d'art), et Bordeaux 3 (collections pédagogiques et de recherche pour l'histoire de l'art et la géographie). Ces fonds, très disparates en quantité et en qualité, connaissent en outre des formes de gestion et de valorisation très inégales.

Mentionnons également pour mémoire le Jardin botanique universitaire de Talence ${ }^{3}$ ainsi que les nombreux bâtiments, dont certains, dispersés au centre ville ou sur les vastes campus de Pessac-Talence et de Carreire, ont une valeur architecturale indéniable. Ils ont fait l'objet de programmes (étude et inventaire raisonné, publications, rénovation, inscription à l'inventaire pour les bâtiments de la place de la Victoire à Bordeaux) qui participent des dynamiques locales de recherche scientifique, de gestion patrimoniale et de valorisation.

\section{Les collections scientifiques de l'université Bordeaux 1}

7 Les importantes collections d'Histoire naturelle et d'instruments scientifiques, issues pour beaucoup des cabinets spécialisés constitués lors de la création du Palais des Facultés à la fin du XIXe siècle, ont été dispersées, pour la plupart dans les années 1960, lors du déménagement du Palais situé en centre ville vers le campus aménagé en périphérie, à Talence ${ }^{4}$. Cependant, à Talence et à Floirac où s'implante un observatoire astronomique à la fin du XIXe siècle, un patrimoine remarquable est constitué pour les be soins de la recherche et de la pédagogie au cours du XXe siècle. Ainsi, neuf collections comprenant 300000 biens couvrent aujourd'hui les principales disciplines de l'université : astronomie, biologie animale, anthropologie, préhistoire, paléontologie, océanographie, géologie et minéralogie. La neuvième collection est celle comportant l'ensemble des ouvrages et périodiques anciens de la bibliothèque universitaire. Parmi les ensembles majeurs, on peut relever le matériel astrophotographique des années 1890 de l'observatoire (seuls 18 observatoires au monde en sont dotés), les collections de faune régionale, l'une des plus importantes collections européennes de moulages de pièces fos siles anthropologiques, des séries expérimentales de taille de roches dures réalisées par les plus grands préhistoriens tailleurs français, de nombreux échantillons types et figurés en paléontologie, des carottes prélevées dans tous les océans du globe, la seule collection complète des série géologiques d'Aquitaine, la collection la plus complète des minéraux des Pyrénées, ou encore les 86 volumes de la bibliothèque de Lavoisier portant son exlibris.

Collections de Géologie, université Bordeaux 1

Vue de la carothèque-lithothèque historique ; les petites boîtes cartonnées contiennent les résidus bruts de forages recueillis selon les profondeurs ; les échantillons de 1600 forages sont ainsi conservés et étudiés (collections de l'UFR 
des Sciences de la Terre et de la Mer).

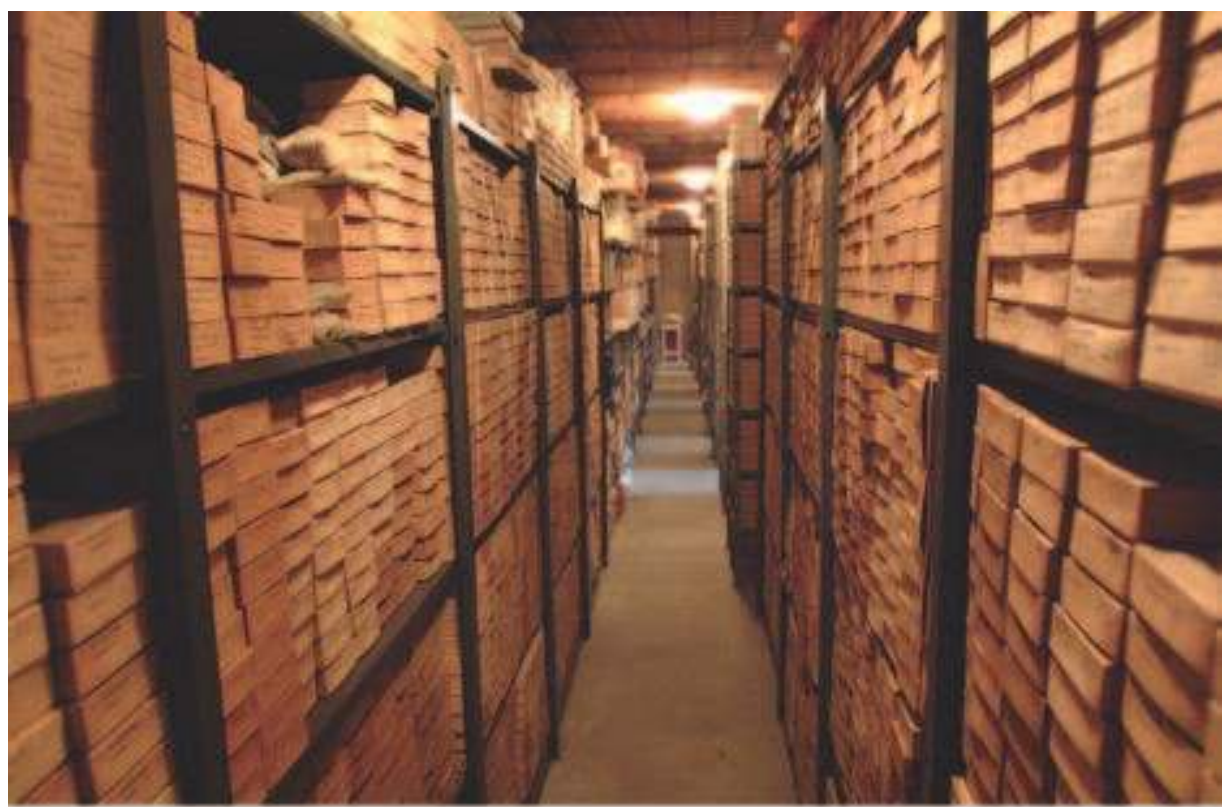

(c) Université Bordeaux 1/Bruno Cahuzac

Carothèque réfrigérée des archives climatiques de l'océan (collections du laboratoire EPOC de l'Observatoire aquitain des Sciences de l'Univers).

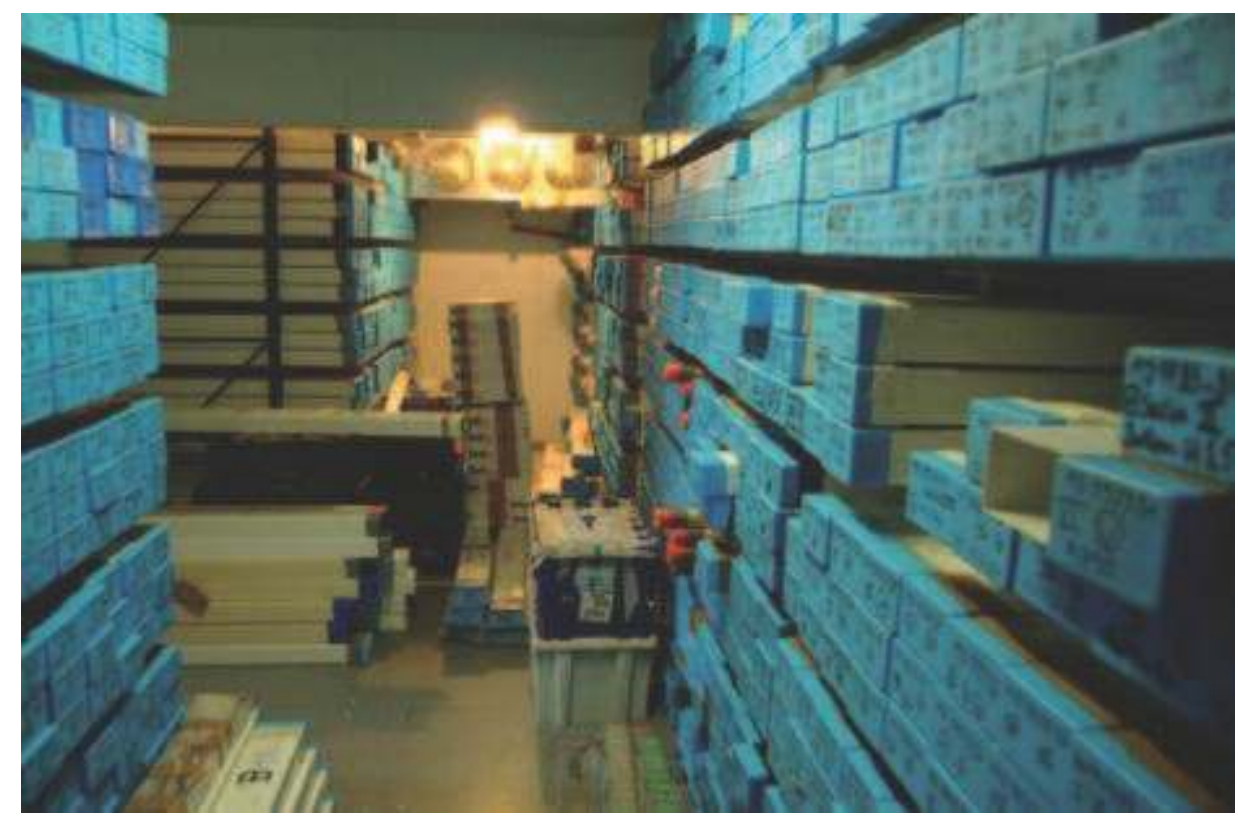

(C) Université Bordeaux 1

8 En ce qui concerne les modalités de conservation de ces collections, plusieurs cas de figure se rencontrent : la majeure partie des collections sont cohérentes, en lien avec leur discipline et regroupées en un seul lieu, même si bien souvent les conditions de conservation ne sont pas optimales; d'autres sont dispersées dans plusieurs sites de stockage ; tandis qu'une partie de la collection de minéralogie a été déposée au muséum d'Histoire naturelle de Bordeaux ${ }^{5}$. Enfin, il faudrait ajouter à ces neuf collections 
clairement identifiées, des ensembles encore laissés à l'abandon dans des caves comme c'est le cas pour les instruments de l'ancien Institut du Pin ou des ensembles en cours de constitution à partir d'items dispersés comme la collection d'appareils d'optique en cours de regroupement.

Ces collections - excepté celles laissées à l'abandon et celles déposées au muséum - sont gérées par des chargés de collection, qui effectuent un travail constant de conservation en plus de leur charge quotidienne de technicien, d'ingénieur ou de maître de con férences. Ils sont cependant soutenus financièrement par la Direction de la Recherche et des Études Doctorales qui attribue chaque année une enveloppe budgétaire. La collégialité des décisions est orchestrée par le vice-président du Conseil scientifique et ce fonctionnement permet de coordonner au mieux les actions. En 2008, une mission d'expertise a été confiée à Mickaël Le Bras, spécialiste du patrimoine scientifique, technique et naturel, afin de dégager les actions prioritaires à mener en termes de conservation préventive ${ }^{6}$. Son étude a permis de cibler les urgences et de faire prendre conscience à la communauté scientifique de Bordeaux 1 de la valeur patrimoniale et scien tifique des collections conservées. Néanmoins, afin de mener les actions préconisées, une politique globale vis-à-vis de ce patrimoine s'avère nécessaire avec le recrutement d'un conservateur pour les piloter. En attendant, on peut citer pour chaque collection de belles actions réalisées sous la houlette des responsables. Par exemple, pour la préhistoire, l'exposition Image d'Ours tenue à la bibliothèque universitaire de sciences et techniques en 2007 a permis de réunir des livres de science du fonds historique de la bibliothèque (Gessner, Aldrovandi...) et des spécimens paléontologiques et zoologiques des collections de l'UMR PACEA. Plus globalement, la bibliothèque universitaire a depuis 2005 une programmation culturelle régulière et participe aux grands événements nationaux (Fête de la Science...) et internationaux (Linné 2007, année mondiale de l'astronomie 2009, année internationale de la chimie 2011...). Les expositions, rencontres, conférences et publications qui animent l'année universitaire et ponctuent la vie de la bibliothèque sont l'occasion de faire découvrir au public les collections patrimoniales documentaires ainsi que les autres collections universitaires bordelaises. Par ailleurs, après avoir inauguré en 2011 un «boudoir scientifique " dédié à la culture scientifique et aux collections d'his toire des sciences et d'épistémologie, la bibliothèque a également ouvert en 2012 une salle d'exposition et d'animation qui en très peu de mois a trouvé son public et constitue un es pace de culture, de convivialité et d'échanges identifié et reconnu.

\section{Les collections de l'université Bordeaux 3}

10 La situation de l'université Bordeaux 3 se présente différemment. Attachées pour l'essentiel à l'enseignement et à la recherche de l'archéologie, de l'histoire de l'art et de la géographie, ses collections sont un héritage de la fin du XIXe siècle et du début du XXe siècle, alors que les sciences humaines prenaient pour exemple les méthodes de recherche et de pédagogie déjà en vigueur chez les scientifiques. Il n'existe pas aujourd'hui de réflexion globale au niveau de l'établissement et ces ensembles sont valorisés par des chargés de collection ou des chercheurs.

11 La cartothèque - tombée dans l'oubli - fait aujourd'hui l'objet d'un inventaire en cours d'informatisation (plus de 20000 cartes), permettant de ce fait la communication auprès des étudiants et des chercheurs. Plusieurs expositions ont été organisées depuis $2010^{7}$. 
Malgré tout, les conditions de conservation demeurent précaires, les locaux n'étant pas adaptés à la préservation d'un tel ensemble.

Le fonds du musée archéologique de l'ancienne faculté des Lettres fut, quant à lui, dispersé au moment où les enseignements se déplacèrent sur le site de Pessac-Talence dans les années 1960. Les moulages et une partie des tirages photographiques ont été mis en dépôt au musée d'Aquitaine. D'autres ensembles sont aussi conservés par le Service commun de Documentation. Si l'intérêt pédagogique ${ }^{8}$ et scientifique de cet ensemble suscite aujourd'hui de nouvelles dynamiques, l'absence d'une réflexion globale sur la conservation donne une perspective précaire à ces initiatives.

Enfin, il est intéressant de mentionner l'existence d'une collection «récente» des données 3D du patrimoine que le Conservatoire numérique 3D ${ }^{9}$ (ARCHEOVISION CNRSBordeaux 3) a pour mission de pérenniser (système d'archivage ARCHEOGRID) ${ }^{10}$.

Moulage de la sépulture de Dolni Vestonice

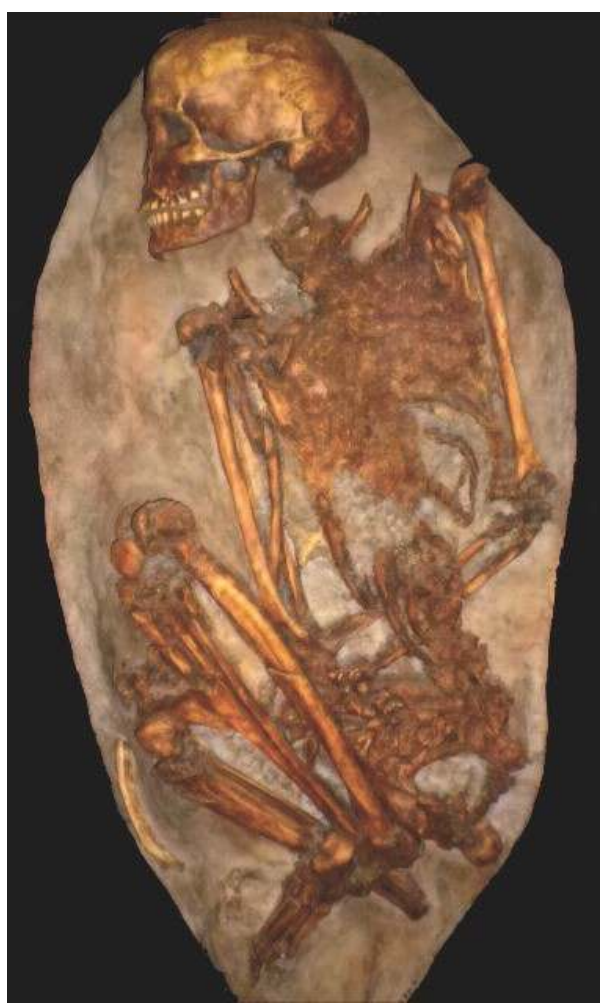

(c) Université Bordeaux 1

\section{Le musée d'Ethnographie de l'université Bordeaux Segalen : un cas atypique}

Le musée d'Ethnographie de l'université Bordeaux Segalen (MEB) est un cas particulier, tant dans le contexte bordelais qu'à l'échelle nationale. Il détient d'importantes collections nationales, héritage des pratiques pédagogiques destinées aux médecins coloniaux (les navalais de l'École de santé de Bordeaux) à la charnière des XIXe et XXe siècles ${ }^{11}$. Ces collections sont les seules à avoir survécu aux vicissitudes du XXe siècle, les autres collections, dont celles de «matière médicale ", de médecine légale ou d'Histoire 
naturelle, ayant été progressivement démontées et, pour certaines, totalement dispersées.

Dans les années 1970-1980, le regroupement des collections ethnographiques, un premier inventaire et des expositions thématiques furent organisés. Depuis 2011, le musée est ouvert au public ${ }^{12}$. Son équipe comprend neuf personnes, dont trois à temps plein et trois emplois-étudiants d'un tiers temps chacun sur neuf mois ${ }^{13}$. Le MEB propose un accès aux collections et à des dossiers documentaires via un site (www.meb.u-bordeaux2.fr) et un blog (http://meb.over-blog.com) régulièrement enrichis, ce qui lui permet de se concentrer sur la diffusion de la recherche et la médiation scientifique, seules missions justifiant qu'une université s'engage dans un projet aussi lourd et coûteux ${ }^{14}$. L'accent a donc été mis sur des expositions temporaires présentant des questions de société croisant documentation ethnographique et éclairage pluridisciplinaire...

Vue de l'exposition Cultiver l'Antique, Archéopôle d'Aquitaine, université Bordeaux 3

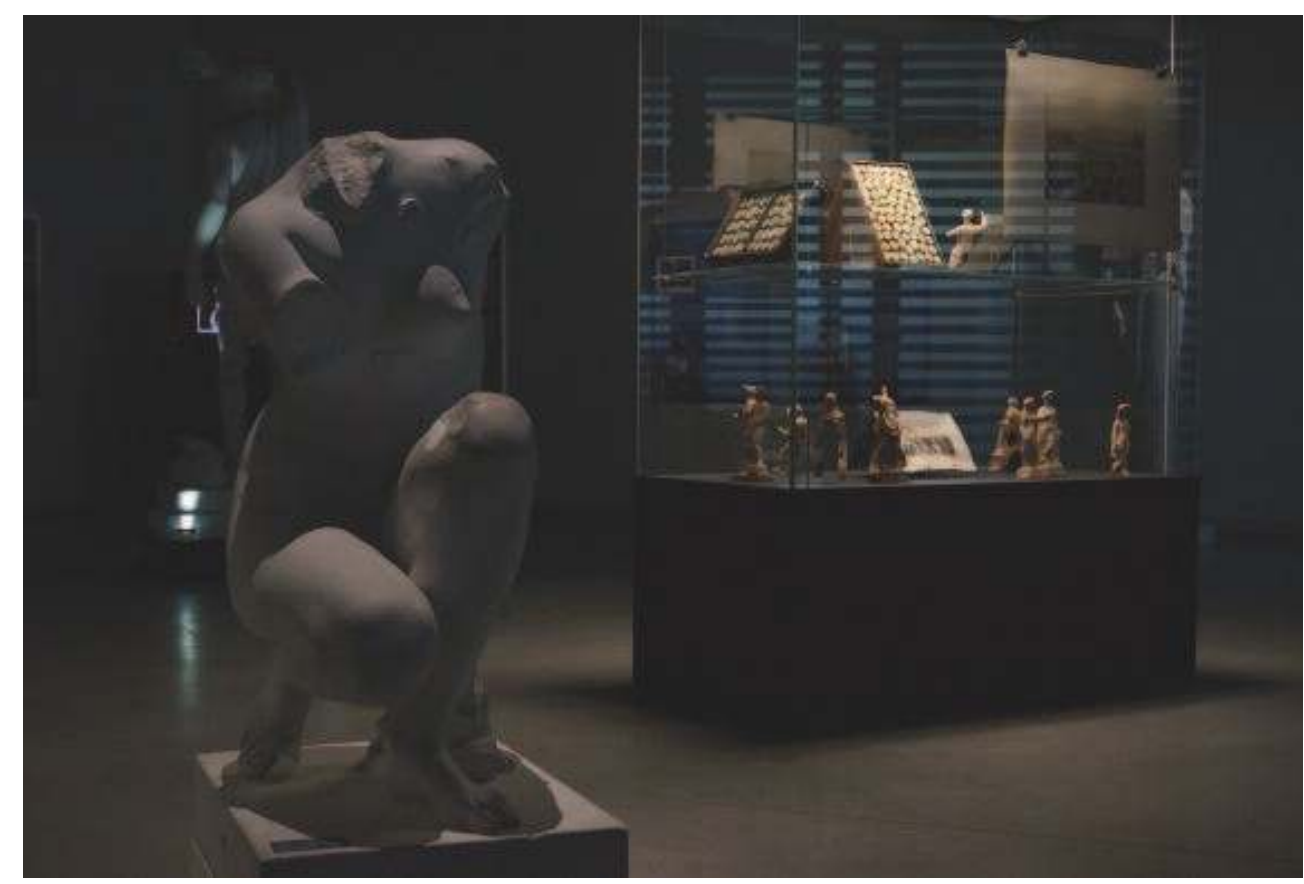

(c) Université Bordeaux 3/Marion Lagrange

Le MEB en est à sa quatrième exposition thématique. La première fut consacrée à sa collection de jonques chinoises anciennes, la deuxième aux mutations culturelles dans le Pacifique, la troisième, intitulée En-Quête de santé, explorait les parcours thérapeutiques et les politiques de la santé. Cette exposition, co-produite avec l'IRD, a bénéficié de nombreux partenariats, notamment avec l'Agence régionale de Santé qui a financé son volet médiation. L'exposition en cours, Paroles d'Afrique, est accompagnée d'un colloque, d'un cycle de conférences, d'une programmation artistique et d'ateliers ouverts aux scolaires. Cette opération, menée en co-production avec le LLACAN (UMR 8135 du CNRS), repose également sur plusieurs partenariats locaux (laboratoires de recherche, associations, collectivités...). Pour la rentrée 2013, une exposition d'art contemporain africain montée en collaboration avec l'Iwalewa Haus (université de Bayreuth) et l'association Migrations Culturelles (MC2A) ouvrira une programmation dense puisque deux expositions (l'une sur la relation entre monnaie et société, l'autre sur l'histoire des 
fonds anciens des bibliothèques universitaires de Bordeaux) se succèderont en moins de huit mois.

Dans ce contexte, le site Internet n'est pas une simple vitrine ou un organe de communication. Développé très tôt pour compenser les difficultés d'accès aux collections, il a vocation à devenir un outil de premier ordre pour une diffusion très large des collections, du savoir et de la recherche en cours. Sa refonte est programmée en lien avec un ambitieux programme de recherche sur la numérisation en trois dimensions des collections textiles (partenariat Manao, INRIA/Institut d'optique).

Précisons enfin que le MEB organise visites pédagogiques, ateliers, conférences et événements culturels. Il accueille des stagiaires, assure des formations, fait visiter ses réserves, participe aux événements annuels (Nuit des Musées, Journées du Patrimoine, Fête de la Science) tout en s'investissant dans la recherche (projets régionaux et ANR). Les compétences acquises, les implications régionales et nationales, sa visibilité dans l'offre culturelle bordelaise, font du MEB un outil de promotion pour l'université bordelaise et, à terme, un outil au service de l'amélioration des conditions de préservation, de gestion et de valorisation des différentes collections du site.

L'université bordelaise se caractérise donc, tout comme les grandes universités de France et d'Europe, par une variété de collections importantes et, plus généralement, par la détention d'un patrimoine dont le potentiel de valorisation reste très fort. Différents pro grammes ambitieux sont en cours, pour mettre en lumière ces collections universitaires et sensibiliser des acteurs locaux à leur intérêt.

Exposition Dynamiques océaniennes proposée dans le cadre de la Nuit des Musées au musée d'Ethnographie de l'université Bordeaux Segalen en mai 2011.

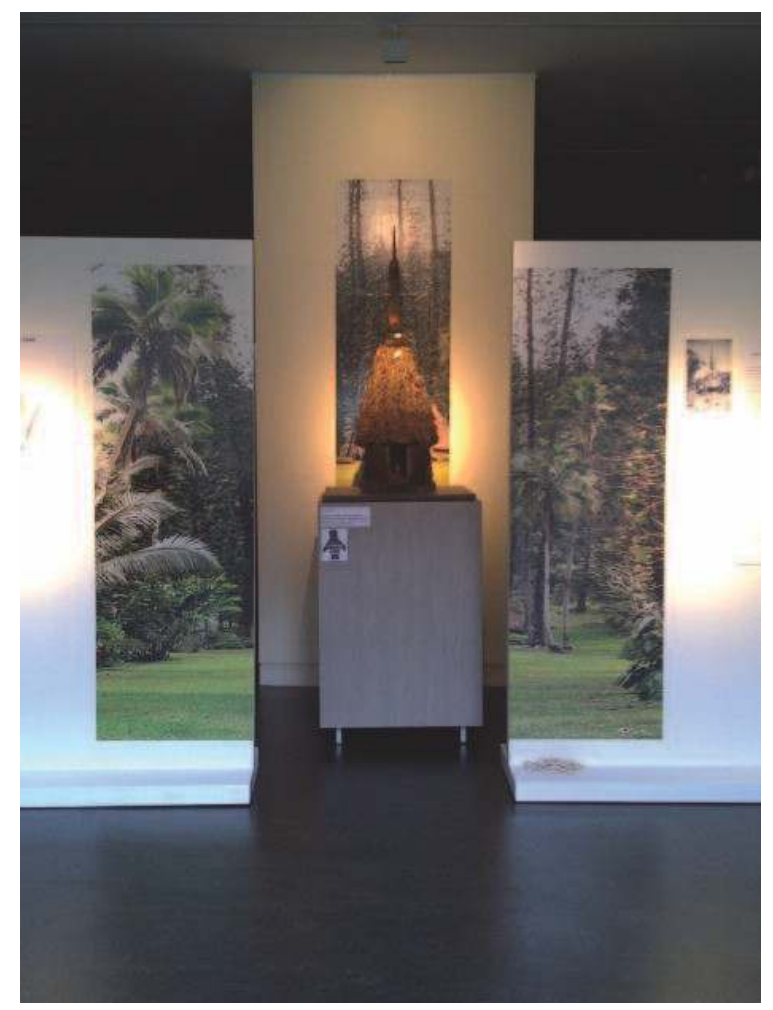

(C) MEB, 2011 


\section{Des dynamiques locales très fortes}

20 leur gestion complexe. Dans la plupart des cas, leur statut est fragile et leur devenir incertain en ce qu'il repose trop largement, comme pour beaucoup de collections universitaires, sur des aspects contextuels (précarité des postes, engagement personnel du directeur, bienveillance de la présidence, bénévolat de salariés...). Ce type de réalisation reste toutefois marginal et fragile tant que des mesures proprement politiques ne soutiennent pas, de façon plus générale, la gestion et la valorisation de leurs collections par les universités qui en sont détentrices, mais aussi, tant que la communauté scientifique ne saura pas défendre des projets de médiation et des axes de patrimonialisation innovants.

21 Les responsables de collections universitaires bordelais travaillent activement à une mise en commun de leurs expériences et à une réflexion commune. Ils ont entrepris une action collective afin d'être force de proposition et de faire reconnaître l'importance de ce pa trimoine scientifique et culturel souvent méconnu, tant auprès des publics que des décideurs, parmi lesquels les ministères de tutelle, la direction du PRES et celle de la future université de Bordeaux.

Dès son arrivée, en 2003, Sophie Chave-Dartoen (Bordeaux Segalen) avait constaté l'absence de toute gestion concertée des collections, de toute politique globale à leur égard, carte blanche lui étant laissée pour le Projet Scientifique et Culturel du musée d'Ethnographie rénové. Un projet de table ronde sur la question émergea de discussions avec Marion Lagrange (maître de conférences en histoire de l'art contemporain Bordeaux 3), projet qui ne se concrétisa pleinement qu'avec l'appui d'un groupe de réflexion organisé à l'époque autour de Jérôme de La Noë, et relayé aujourd'hui par Lætitia Maison-Soulard (Bordeaux 1), tous deux très engagés dans la sauvegarde de l'observatoire de Floirac, menacé de fermeture ${ }^{15}$.

Certaines collections d'Histoire naturelle paraissaient alors gravement en danger, et la mobilisation fut générale afin de constituer un collectif capable de proposer un état des lieux et des propositions concrètes qui puissent être intégrées dans le projet Campus, alors à ses débuts. 


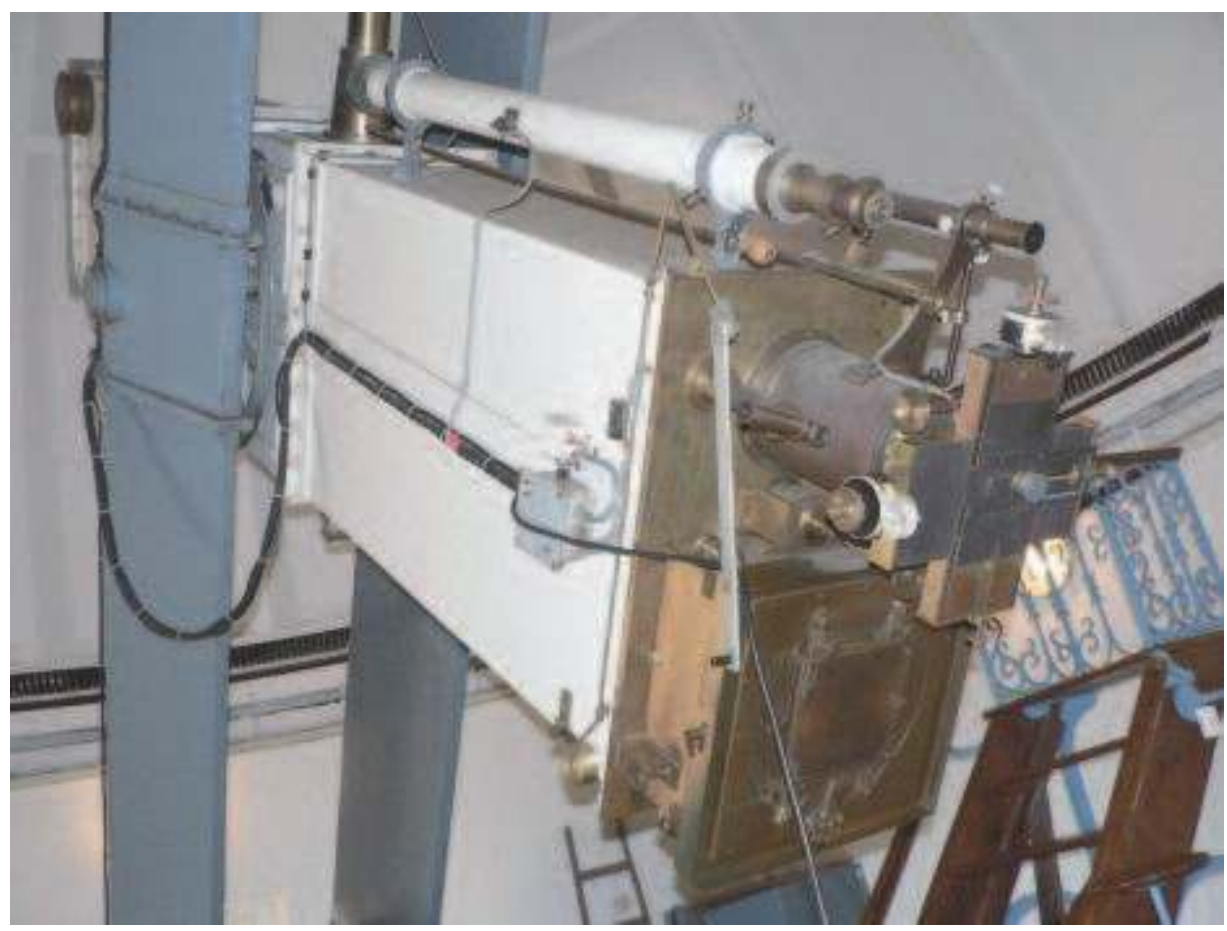

(C) Université Bordeaux 1 Ces journées permirent la formalisation d'un premier état des lieux, une identification des ressources disponibles et une sensibilisation des tutelles aux questions de sauvegarde et de gestion des collections universitaires. En parallèle, le collectif œuvra pour la réalisation d'une exposition commune (voir ci-après) et à la création de programmes de recherche en histoire de l'art et en histoire des sciences autour des collections.

\section{La réalisation d'une exposition commune au CCSTI de Bordeaux, Cap Sciences : l'occasion d'unir nos forces}

Le projet d'une exposition des collections universitaires est né dès 2009 entre les responsables du CCSTI Cap Sciences et les responsables de collections, tout d'abord de l'université Bordeaux 1 détentrice d'environ 300000 objets sur les 500000 recensés sur l'ensemble du campus universitaire. Très rapidement et dans l'optique de la NUB, il a paru indispensable d'étendre le sujet de l'exposition à l'ensemble des collections universitaires afin de poursuivre les réflexions du collectif interuniversitaire. L'équipe de Cap Sciences a donc invité la Commission interuniversitaire à travailler sur les différents thèmes d'une exposition de synthèse, présentant l'ensemble des collections et intitulée Motif(s) de collection ${ }^{17}$. 
Page d'accueil des collections de l'UFR de biologie animale, site Internet de l'université Bordeaux 1

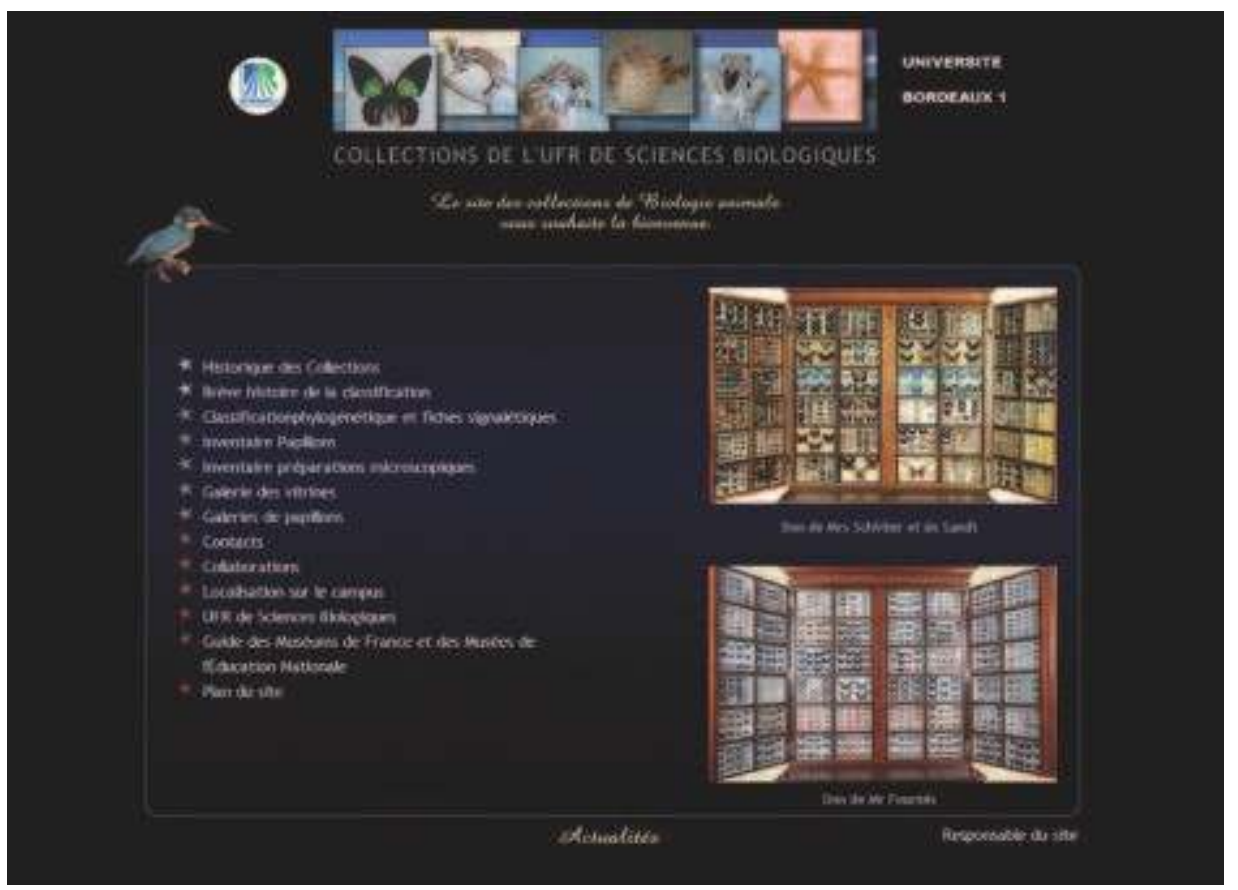

(c) Université Bordeaux 1

27 Cette exposition a ainsi été l'occasion de renforcer les liens au sein du collectif interuniversitaire et de travailler sur les arguments à faire valoir pour continuer à préserver les collections universitaires. En s'interrogeant sur les pratiques savantes qui ont conduit à la formation du patrimoine universitaire, les responsables de collections ont pu formuler les raisons pour lesquelles il est primordial pour eux de continuer à travailler sur les anciennes collections ou d'en constituer de nouvelles. Si certaines collections ont encore un rôle majeur en pédagogie ou en recherche, l'exposition a permis aux membres du collectif d'établir et de formaliser les convergences, voire les points communs qu'elles présentent. L'un d'eux, essentiel, est la possibilité de regarder chacune d'elles avec les yeux de l'historien des sciences et de lui faire raconter des histoires sur les pratiques savantes et les avancées dans la connaissance scientifique. En un mot, ces collections sont des objets culturels.

28 Nous voici donc justement au cœur du processus de patrimonialisation décrit par Jean Davallon dans Patrimoine et Communautés savantes, paru en 2009 : " La recontextualisation qu'opère la démarche patrimoniale sur les objets ou des connaissances conduit à se demander si une histoire culturelle des sciences n'est pas la condition sine qua non pour rendre ces objets ou ces connaissances patrimoniales. [...] Ce qui signifie que, pour être opérante, la patrimonialisation de l'activité scientifique et de ses objets suppose le regard d'une science réflexive qui produise une connaissance de l'ob jet et de l'activité scientifique d'où il vient $»^{18}$.

29 Ainsi, par le caractère synthétique de l'exposition nécessitant de présenter ces collections par le biais de ce qui les unit, à savoir les pratiques savantes de collecte et d'étude, nous avons développé une approche épistémologique pour les présenter aux publics, ce qui a permis en retour un pas de plus vers leur patrimonialisation. 
30 Il faut noter, pour conclure sur ce point, que d'autres efforts de valorisation du même type seront conduits à l'avenir : ainsi, une exposition sur l'ensemble des collections des bibliothèques universitaires sera présentée au musée d'Ethnographie de Bordeaux au printemps 2014.

\section{Des programmes de recherche épistémologiques comme usage complémentaire des collections}

31 Un problème général aux collections universitaires est qu'elles ne sont plus, de nos jours, aussi cruciales pour l'enseignement comparé au moment de leur création. Toutes ne sont pas, non plus, objets de recherche direct ou instruments de recherche. Et beaucoup restent dans les laboratoires et dans les caves comme les témoins de pratiques anciennes et obsolètes. Un tel regard oublie toutefois l'actualité des questions qu'elles posent à l'histoire de chacune des disciplines impliquées, d'une part, et de façon plus générale, à l'histoire et à la philosophie des sciences. Aussi toutes ces collections ne sont pas en déshérence et, si certaines sont des objets quotidiens de recherche (anthropologie physique, ethnographie, océanographie...), d'autres offrent des supports de réflexion et de travaux dans le cadre des masters professionnalisant proposés par les universités Bordeaux 1 et 3 , ainsi que dans le cadre de thèses de doctorat ${ }^{19}$.

Outre le support qu'elles offrent à des formations et à des recherches spécifiques, les collections bordelaises font l'objet de plusieurs programmes de recherche ambitieux coordonnés par les universités.

Le premier d'entre eux (2010-2013) s'attache à la localisation, à l'inventaire et à la documentation du patrimoine artistique de l'université bordelaise dans son ensemble (fin XIXe siècle-années 1960). Ce programme se définit en fonction d'une approche spécifique aux historiens de l'art, en rapport avec ses objets d'étude (beaux-arts, architecture, arts décoratifs, photographie...), tout en n'excluant pas des rapprochements interdisciplinaires, essentiels dans le cadre d'une telle réflexion. L'attention s'est notamment portée sur l'ancien musée archéologique dont les collections servaient à l'enseignement de l'histoire de l'art et de l'archéologie (près de 500 moulages et 4000 photographies). Outre l'inventaire, le programme a notamment permis de mettre en place une activité de numérisation très importante, à la fois pour les moulages et pour les photographies, objets existants mais difficilement accessibles. Une partie des données re cueillies lors de l'inventaire est d'ores et déjà disponible par le biais de la bibliothèque nu mérique conçue et mise en ligne par le SCD de Bordeaux $3^{20}$ et d'un site d'expositions virtuelles ${ }^{21}$. Ce programme donne, ou pourra donner lieu à des recherches universitaires, dans le domaine de l'histoire de l'art (Les collections universitaires de moulages, thèse en cours de Soline Morinière, sous la direction de Dominique Jarrassé), de l'histoire de la photographie (La collection photographique d'histoire de l'art antique de la faculté des lettres. La photographie de sculpture antique, représentation et pédagogie, mémoire de Master 1 de Pierre Garganette, sous la direction de Marion Lagrange). Outre ce travail scientifique, des actions de valorisation, grand public mais aussi pédagogiques, pourraient être envisagées dans le domaine de la réalité augmentée.

Le patrimoine mobilier fait l'objet d'un second projet interdisciplinaire centré sur l'inventaire de l'instrumentation contemporaine scientifique et technique (2011-2014). Co-financé par la région Aquitaine et par la mission nationale PATSTEC, il est coordonné par Lætitia Maison-Soulard et réunit des historiens des sciences, des historiens de la 
période contemporaine et des professionnels de l'inventaire et de la médiation scientifique $^{22}$. Ce projet vise à recenser et à valoriser le patrimoine scientifique, technique et industriel aquitain, notamment dans le domaine aéronautique. Nombreux sont, en effet, les laboratoires du campus ayant acquis et mis au point une instrumentation dédiée à des projets en lien avec l'industrie aéronautique depuis les années 1960. Ce corpus constitue un témoignage complémentaire aux sources archivistiques sur l'histoire des pratiques savantes relatives à un sujet particulièrement connecté au complexe militaroindustriel girondin et qui ouvre des points de comparaison avec la région Midi-Pyrénées par exemple, dont l'histoire concerne davantage l'aéronautique civile. Les données sont en cours de saisie dans la base PATSTEC et la base GERTRUDE du Service régional de l'Inventaire.

Le troisième projet s'intéresse au patrimoine documentaire universitaire bordelais et fait également l'objet d'attentions conjointes. Piloté également par la Maison des Sciences de l'Homme d'Aquitaine (MSHA) et la bibliothèque universitaire de Bordeaux 1 , le programme s'intitule "La bibliothèque numérique Uranie » et a pour ambition de valoriser les fonds d'ouvrages scientifiques conservés dans les bibliothèques municipales et universitaires d'Aquitaine. En relation avec la recherche, elle est le prolongement du programme de la MSHA : «Le livre scientifique : définition et émergence d'un genre (1450-1850) » qui s'est déroulé de 2007 à 2010.

L'idée de la création d'Uranie est née d'un double constat: d'une part la grande richesse des fonds scientifiques anciens des bibliothèques bordelaises, fonds méconnus et négligés, qui méritent que l'on assure leur promotion, leur diffusion et leur protection par la numérisation et d'autre part l'absence d'un inventaire général. Outre son intérêt patrimonial, un tel inventaire permet désormais d'identifier divers foyers d'activité scientifique, de mieux comprendre le rôle des sociétés savantes et des différents types de bibliothèques et de voir se dessiner une structure de l'histoire de la diffusion des savoirs en Aquitaine ainsi que le rôle des universitaires au XIXe siècle. 
Droguier du bureau du jardinier (université Bordeaux Segalen) : de nombreux bocaux qui faisaient partie d'un ancien musée de Matière médicale sont entreposés dans l'orangerie du Jardin botanique de Talence.

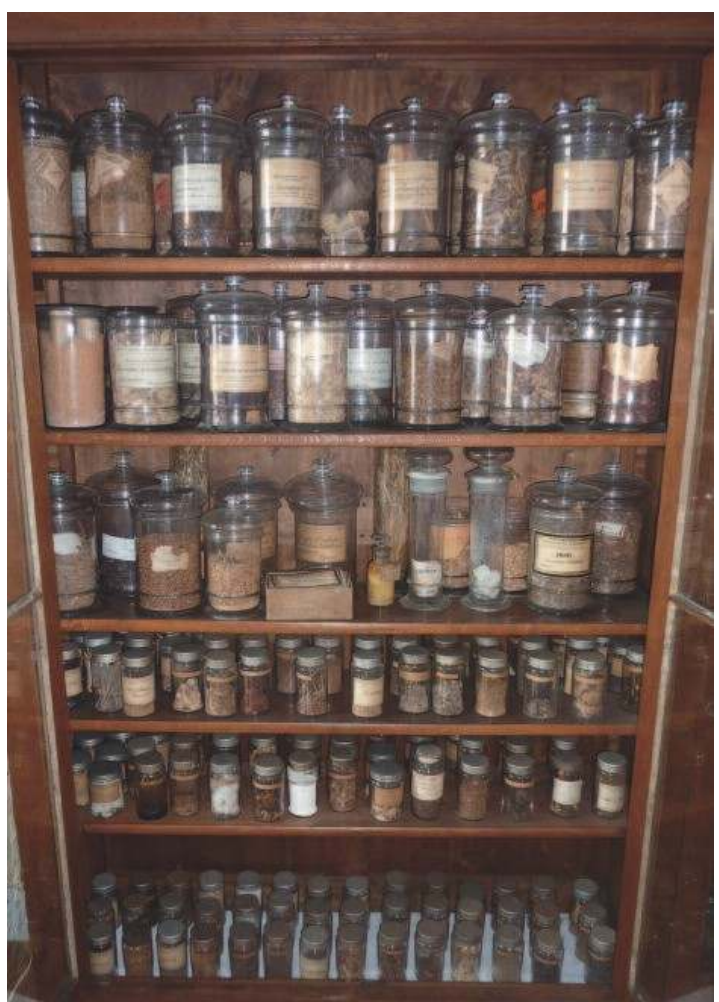

(c) Université Bordeaux Segalen/Alain Badoc 
Herbier Tempère de 30 liasses sur la flore française en bon état et de grand intérêt scientifique, conservé dans le bâtiment d'enseignement du Jardin botanique de Talence (université Bordeaux Segalen). Gaston Tempère (1900-1985) fut chef de travaux pratiques au laboratoire de Botanique et Matière médicale de la faculté de Médecine et Pharmacie de Bordeaux.

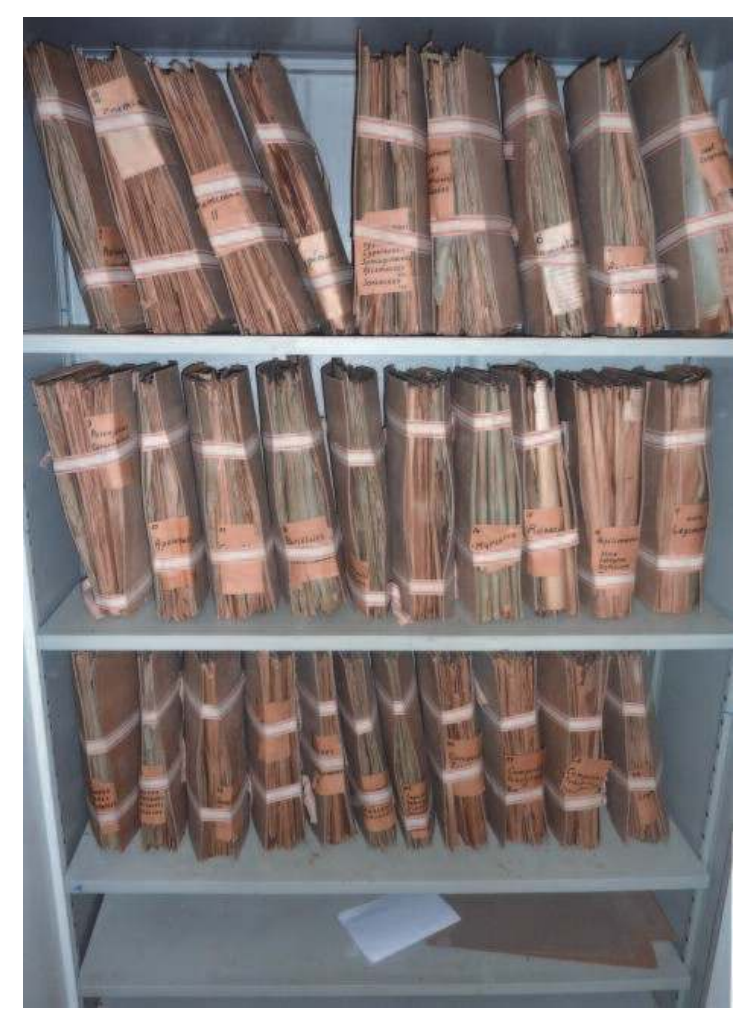

(c) Université Bordeaux Segalen/Alain Badoc

Son corpus actuellement limité aux livres anciens d'astronomie, est révélateur d'une évolution épistémologique et intellectuelle et témoigne de l'histoire du livre scientifique avec deux ensembles originaux : les incunables d'astronomie, qui correspondent à la première Renaissance de l'astronomie (1450-1500), et les manuels de pratique astronomique (XVIe-XIXe siècles) notamment avec des ouvrages en langue française et des livres d'usage.

Uranie est désormais enrichie par le programme de recherche « Formes du savoir de 1400 à 1740 » de la MSHA et devrait être accessible au public courant 2013.

Un dernier projet, animé par le département Documentation du PRES de l'université de Bordeaux, concerne le classement et l'inventaire des manuscrits et archives des bibliothèques universitaires: soutenue en partie par l'Agence bibliographique de l'En seignement supérieur, la mise à jour des données de la base nationale CALAMES $^{23}$ a été profondément renouvelée en 2012, permettant d'intégrer des collections non traitées jusqu'alors (par exemple : les enquêtes linguistiques de J. Saroïhandy ou les archives de l'Association radiophonique de la Côte d'Argent conservées à la bibliothèque universitaire de Bordeaux 3, les fonds de manuscrits de Jacques Ellul et Bernard Charbonneau conservés à la bibliothèque de Sciences-Po Bordeaux). Ce travail se poursuivra en 2013-2014 par l'intégration de collections scientifiques très importantes pour l'histoire de l'université : rétroconversion de l'inventaire des archives de l'observatoire de Floirac, classement de divers manuscrits de scientifiques issus de laboratoires du campus. 
40 Forts de ces actions interdisciplinaires qui font émerger le patrimoine universitaire bordelais dans des projets de recherche, des publications et des actions pédagogiques et culturelles - notamment celles portées par le MEB -, nous avons, depuis 2011, concentré notre travail sur une dimension plus proprement politique du dossier et sur la mobilisation des tutelles, tant locales que nationales.

\section{Plan campus et démarches auprès du PRES}

41 La réflexion collective sur l'avenir des collections universitaires, impulsée lors de colloques nationaux successifs ${ }^{24}$, a été menée, ces dernières années, à deux échelles. La première, nationale, a vu une mobilisation des responsables de collections qui s'est ré cemment concrétisée dans deux réalisations. L'une d'elle est la Plateforme OCIMUniversités qui vise au recensement des collections et des actions menées par les universités françaises dans le domaine de la culture scientifique et technique. L'autre est le texte, initialement pensé comme un manifeste, préparé par un comité national informel et discuté lors du symposium de Strasbourg ${ }^{25}$. Ce texte, présenté dans le cadre des Assises nationales de la Recherche, demande une politique active de l'État et des tutelles en soulignant que les collections participent à la recherche et à sa valorisation, qu'elles offrent une ressource pour la transmission des savoirs à l'université, qu'elles constituent un médium essentiel pour intéresser les citoyens aux missions des universités et que, tout en étant vulnérables, elles sont un outil de prestige, mais aussi de stabilité en ce qu'elles ancrent savoirs et pratiques scientifiques dans la durée ${ }^{26}$.

42 À l'échelle locale, des actions concertées on été menées parallèlement. Suite à notre demande, le collectif bordelais été invité par le bureau du PRES à présenter un état des lieux et un projet global. 
Page d'accueil du site des expositions virtuelles sur le patrimoine artistique de l'université de Bordeaux

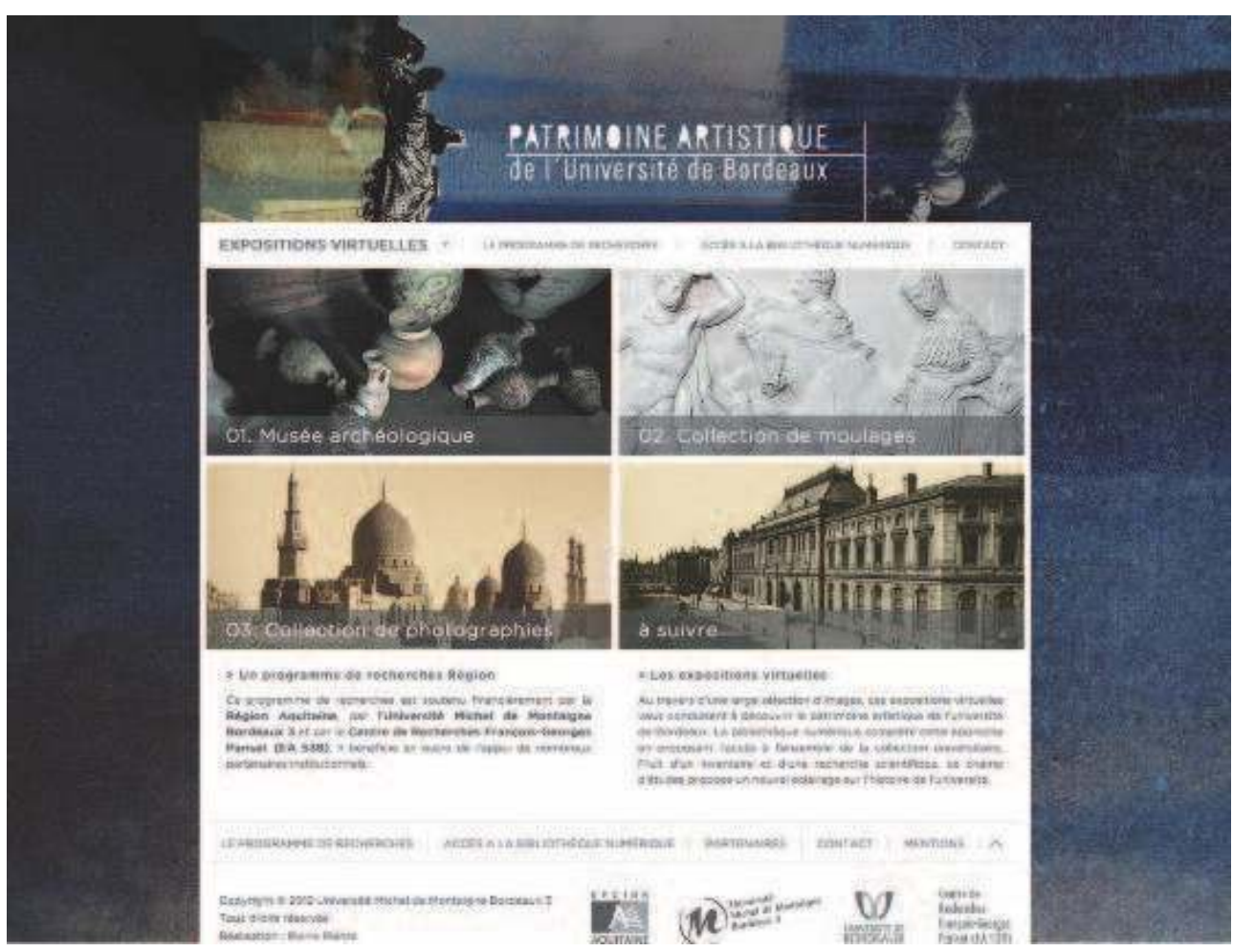

(C) Université Bordeaux 3/Marion Lagrange

43 La préparation de cet exposé fut collective et a débouché sur la préparation d'un document de synthèse. Le programme proposé est pragmatique : il s'appuie sur l'existant et de façon progressive, permettrait de remplir certains des objectifs affichés par le PRES actuel et la future NUB. Il repose sur une idée forte que nous avons défendue avec con viction : la connaissance partagée d'un patrimoine commun pourrait contribuer à renforcer le sentiment d'une communauté où les distinctions (université/cité, enseignants-chercheurs/étudiants, sciences et techniques/sciences humaines et sociales...) seraient transcendées; l'idée en somme qu'une valorisation judicieuse des collections universitaires pourrait contribuer à la mise en évidence d'une histoire, de pratiques et de valeurs communes, et ainsi permettre l'émergence d'un " esprit de campus » fédérateur pour des structures en cours de rapprochement, mais aussi pour la communauté universitaire ainsi recomposée ${ }^{27}$.

Nous avons donc proposé une structure légère mais intellectuellement intégrative telle une plateforme scientifique et culturelle déclinant le programme en trois volets: un portail Web, une offre culturelle et un parcours entre bâtiments et collections, à travers la ville et le campus. Ce projet, très léger, demande de réajuster certains espaces sans en créer de nouveaux. Les objectifs principaux seraient ainsi de rendre les collections accessibles, tout au moins par la numérisation et la diffusion en ligne afin d'en permettre une exploitation scientifique optimale, et de rendre toute sa place à l'université au sein de la cité en proposant une offre en matière de culture scientifique et de diffusion de la recherche pour tous les publics. Le musée d'Ethnographie de l'université Bordeaux Segalen pourrait être ici une ressource et un rouage central de cette nouvelle structure, mais des moyens ont été demandés pour le pilotage général (un poste d'ingénieur de 
recherche attaché au PRES) et, pour l'essentiel du budget, la création d'un site et la mise en ligne des collections.

Pour conclure, nous souhaitons affirmer que conservation et valorisation des collections reposent nécessairement sur deux formes de légitimité. La première est épistémologique : les collections doivent fonder l'histoire des pratiques scientifiques et une réflexion de fond sur la construction et la diffusion des savoirs. La seconde est politique, en ce que les collections et leur valorisation peuvent être mises au service d'une définition des universités françaises et de leurs identités spécifiques (histoire, spécificités disciplinaires, ancrage local...), dans la mesure où une telle mission est affichée et soutenue par la politique de l'université.

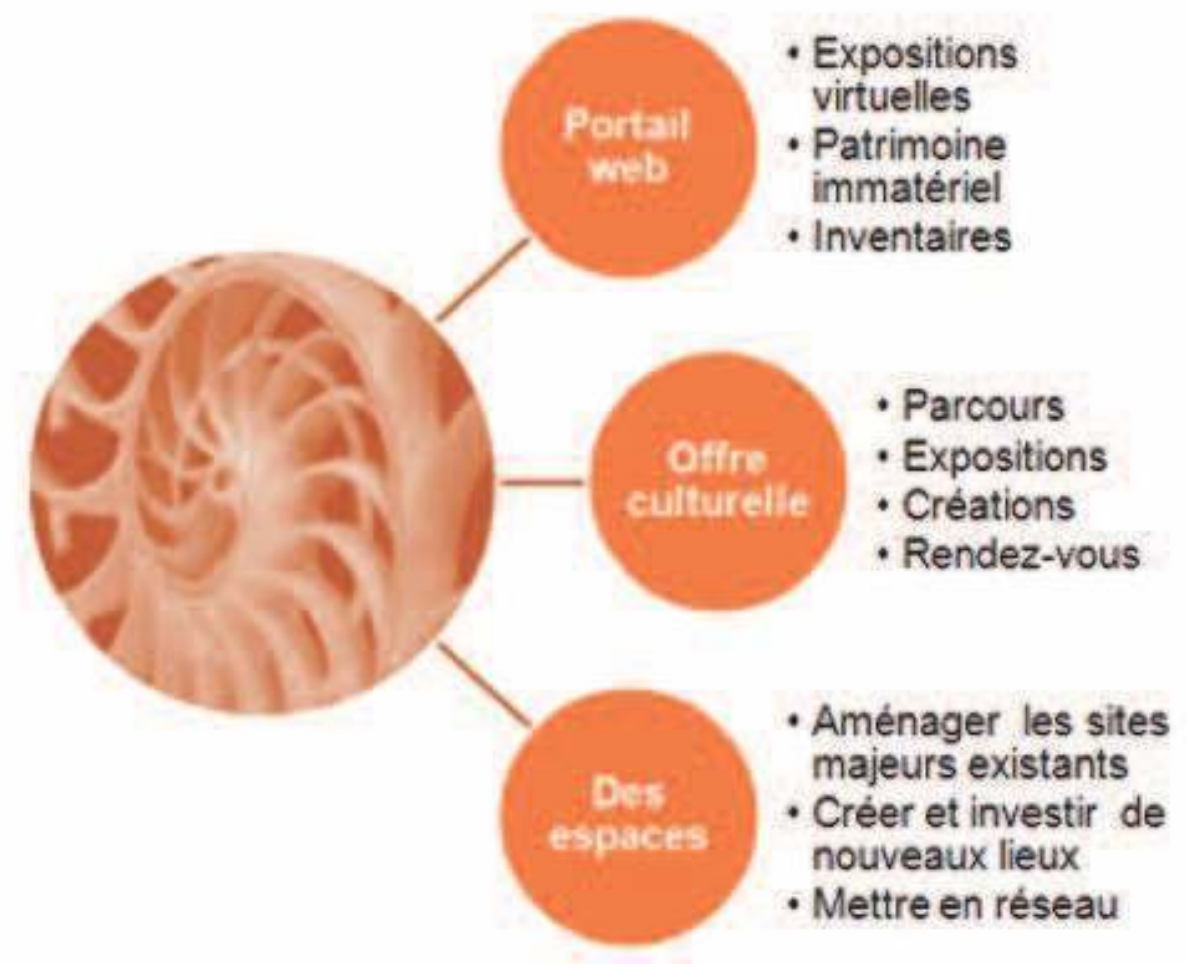

Nous insistons donc sur le fait que ce patrimoine ne peut servir l'image de l'université que si le regard porté sur lui intègre la dimension historique et réflexive permettant de considérer ces collections comme un témoignage des pratiques sociales et savantes, et de leur conférer en retour une forme de «légitimité patrimoniale ». L'enjeu est ici à la fois local et national car il demande un soutien à long terme de la part des institutions de tutelle et des instances d'évaluation qui accompagnent la mise en place de politiques publiques.

\section{NOTES}

1. Sanz, N. et Bergan, S. Le patrimoine des universités européennes. Strasbourg: Éditions du Conseil de l'Europe, 2006. Regards sur le patrimoine scientifique des université, La Lettre de l'OCIM 
, $\mathrm{n}^{\circ}$ 129, mai-juin 2010; voir aussi les travaux de UMAC au sein de l'ICOM (http:// publicus.culture.hu-berlin.de/umac/) et de l'association européenne UNIVERSEUM (www.universeum.it/).

2. L'université Michel de Montaigne Bordeaux 3 dédiée aux humanités, jusqu'à présent restée à l'écart du processus, annonce un référendum sur le sujet pour le printemps 2013. En revanche, l'Institut d'Études Politiques (IEP-Sciences Po Bordeaux) et l'Institut Polytechnique de Bordeaux (IPB), un temps pressentis pour intégrer la NUB, n'y fusionneront pas.

3. Une partie des herbiers est en effet conservée au Jardin botanique universitaire de Talence (dépendant de Bordeaux Segalen) qui présente également plus de 1700 espèces vivantes. Le reste des herbiers a été déposé au Jardin botanique de Bordeaux.

4. On peut également mentionner la dispersion des collections conservées au «musée de pathologie exotique et d'études coloniales » créé à la faculté mixte de Médecine et de Pharmacie en fin de XIXe siècle, musée dont ne survivent que les collections ethnographiques de l'actuel MEB.

5. C'est le cas également des collections d'herpétologie de Bordeaux Segalen.

6. Aujourd'hui conservateur du muséum de Clermont-Ferrand, et spécialiste du patrimoine scientifique, technique et naturel.

7. Le bassin d'Arcachon à travers les cartes ; 2011 : Lignes de côte. Les mutations du littoral du Sud-Ouest de la France depuis le XVIIe siècle; 2012: L'eau dans notre vie et notre environnement.

8. Cultiver l'Antique, Pessac, Archéopôle d'Aquitaine, exposition du 6 mars au 26 avril 2013.

9. http://aecheovision.cnrs.fr/spip.php ?rubrique40

10. http://archeogrid.in2p3.fr

11. L'université Bordeaux Segalen détient en outre des collections botaniques (graines et herbiers), des instruments scientifiques (odontologie par exemple) et quelques œuvres d'art, dont une remarquable fresque d'André Lhote. Mentionnons que les bâtiments du site Victoire viennent d'être inscrits à l'inventaire des Monuments historiques.

12. La rénovation du MEB résulte d'un projet de grande ampleur inséré dans le cadre de deux contrats de plan État-Région. Pour le chantier des collections, le travail de M-D. Parchas (2005), stagiaire de la MST de Paris 1 "conservation préventive ", l'établissement d'un Projet Scientifi que et Culturel chiffré (S. Chave-Dartoen, 2005) et d'un Cahier des charges pour la rénovation des bâtiments (S. Chave-Dartoen, M.-D. Parchas, B. Sansamat, 2005) permirent d'obtenir du ministère de l'Enseignement supérieur et de la Recherche une enveloppe globale de 160000 .. Des spécialistes de conservation préventive purent ainsi être recrutés pour le pilotage des différentes opérations.

13. L'équipe comprend un MCF ( 60 heures de décharge) et un IR $30 \%$. Pour les autres (une personne à temps plein et une à temps partiel sur CDI, deux sur CDD temps plein), l'université finance les postes.

14. L'idée d'une exposition permanente a été écartée, faute d'espaces suffisants ( $250 \mathrm{~m}^{2}$ environ), mais aussi du fait de la proximité du musée d'Aquitaine dont les collections exotiques sont remarquables.

15. Après une thèse sur l'observatoire astronomique de Bordeaux et cinq années passées en tant qu'attachée de conservation au musée des Confluences de Lyon, Lætitia Maison-Soulard est aujourd'hui chargée de mission par Bordeaux 1 pour les collections universitaires tout en étant détachée sur un post-doctorat au sein de l'équipe d'accueil Sciences, Philosophie, Humanités cohabilitée par les universités Bordeaux 1 et Bordeaux 3 , afin de mener un projet régional d'inventaire du patrimoine industriel, scientifique et technique en Aquitaine pour 3 ans (2011-2014).

16. Séminaire des collections universitaires de Bordeaux, les 29 et 30 juin 2010, Domaine du HautCarré, Bordeaux 1. 
17. Ouverte du 2 mai au 18 septembre 2011 à Cap Sciences (Bordeaux) cette exposition proposait une visite organisée autour de trois grandes questions: qui collectionne quoi? Pourquoi collectionner? Quelle relation entre recherche et collection? Des parcours adaptés et des ateliers étaient proposés à des publics spécifiques. Cette exposition a reçu 10000 visiteurs et un bon accueil de la presse locale malgré sa courte durée et la césure de la période estivale.

18. Davallon, J. postface à l'ouvrage dirigé par Boudia, S., Rasmussen, A. et Soubiran, S. Patrimoine et Communautés savantes. Rennes : PUR, 2009, 297 p.

19. Du point de vue de la recherche en histoire des sciences, elle est effectuée aujourd'hui au sein du laboratoire Sciences, Philosophie, Humanités co-habilité par Bordeaux 1 et Bordeaux 3. La première thèse de doctorat en histoire des sciences a justement concerné une collection, celle de l'astronomie: Maison, L. La fondation et les premiers travaux de l'observatoire astronomique de Bordeaux (1871-1906) : histoire d'une réorientation scientifique. Thèse de l'université Bordeaux 1, 2004. Les étudiants sont également amenés à travailler sur les collections au sein du Master HPMS (Histoire, Philosophie et Médiations des sciences) cohabilité par les universités Bordeaux 1, Bordeaux 2, Bordeaux 3, et par l'IEP SciencePo Bordeaux.

20. http://1886.u-bordeaux3.fr/

21. http://patrimoine-artistique.u-bordeaux3.fr/index.php

22. Voir le blog de l'enquête: www.industriel-patrimoine-aquitaine.fr et le site PATSTEC: www.patstec.fr/PSETT

23. CALAMES est le catalogue collectif national des archives et manuscrits des bibliothèques de l'Enseignement supérieur et de la Recherche

24. Patrimoine culturel scientifique et technique des universités, 5 mars 2004, université de Lille ; Les collections universitaires. Journées nationales de réflexion et d'étude sur le Patrimoine scientifique des universités, co-organisées par le ministère de l'Éducation nationale, de l'Enseignement supérieur et de la Recherche (Direction de la Recherche) et le Pôle universitaire européen de Montpellier et du Languedoc-Roussillon, 18 et 19 novembre 2004 ; Gestion et mise en public des collections universitaires, symposium international organisé par le Jardin des sciences de l'université de Strasbourg et le LAMIC de l'université de Laval à Québec, 26 et 27 février 2009, Strasbourg.

25. Pour un réseau national des musées et collections universitaires, journées d'étude organisées par le Jardin des Sciences de Strasbourg en partenariat avec l'ociM-université de Bourgogne, 2 et 3 février 2012.

26. Pour l'intégration des musées et collections dans la politique scientifique et culturelle des universités. Manifeste pour une action nationale concertée, accessible en ligne: www.assises.esr.fr/var/ assises/storage/original/application/370b47d7d7ce7c1897e4c15c73d6e893.pdf

27. Nos inspirations ont été clairement citées : Oxford, Strasbourg, mais aussi le Massachusetts Instite of Technology (MIT) ou Cambridge.

\section{RÉSUMÉS}

Après avoir dressé un panorama des collections scientifiques des universités bordelaises et à partir de la réflexion collective menée sur leur avenir et leurs enjeux politiques et culturels, les auteurs décrivent le long processus de patrimonialisation de ces collections et montrent 
l'importance de leur valorisation comme élément fédérateur de la nouvelle université de Bordeaux.

INDEX

Mots-clés : musée technique, patrimoine industriel, réseau

\section{AUTEURS}

SOPHIE CHAVE-DARTOEN

Sophie Chave-Dartoen est directrice du musée d'Ethnographie de l'université Bordeaux Segalen. sophie.chave-dartoen@u-bordeaux2.fr

\section{LAETITIA MAISON-SOULARD}

Lætitia Maison-Soulard est attachée de conservation du patrimoine, docteur en histoire des sciences, chargée de mission collections universitaires à l'université Bordeaux1. laetitia.maison@msha.fr

\section{MARION LAGRANGE}

Marion Lagrange est maître de conférences à l'université Michel de Montaigne Bordeaux 3 lagrange.marion@gmail.com 